\title{
Protoplanetary Disk Evolution and Influence of the Host Star
}

\author{
Kévin Baillié and Sébastien Charnoz \\ Laboratoire AIM, Université Paris Diderot / CEA / CNRS \\ 91191 Gif-sur-Yvette, France \\ email: kevin.baillie@cea.fr
}

\begin{abstract}
Based on a self-consistent coupling between protoplanetary disk thermodynamics, photosphere geometry and dynamics we designed a 1D-hydrodynamical numerical model for the spreading of the disks as a function of the star characteristics. We found that the evolution timescale increases for more massive or for a steeper surface density disk, and decreases for bigger stars or less turbulent disks. We found a strong dependency of the mass accretion rate versus the disk mass and a weaker dependency versus the star mass. Coupled with observed similar conclusions, we derived that the disk mass is scaling as $M_{*}^{1.6}$.
\end{abstract}

Keywords. planetary systems: protoplanetary disks, hydrodynamics, methods: numerical

\section{Introduction}

Recurrent observations (Andrews et al. 2009; Isella et al. 2009) in non-genetically related regions provide the following constraints:

- Surface-mass density : $\Sigma(r) \propto r^{-q}$ with $q \sim 1$

- Disk photosphere geometries are flared : $H_{\text {photo }}(r) \propto r^{1.1-1.3}$

- Mass accretion rates : $\dot{M} \sim 10^{-8}-10^{-6} \mathrm{M}_{\odot} / \mathrm{yr}$

We present our results of numerical simulations of the evolution of protoplanetary disks on large scales in order to explain the origins of these common properties.

\section{Methods}

We have designed a 1D-hydrodynamical code that takes into consideration

- a coupling between dynamics and thermodynamics through the turbulent viscosity $\nu=\alpha_{\mathrm{visc}} c_{\mathrm{s}} h_{\mathrm{pr}}$, where $\alpha_{\mathrm{visc}}$ is the viscosity prescription from Shakura \& Sunyaev (1973), $c_{\mathrm{s}}$ is the local sound speed and $h_{\mathrm{pr}}$ is the pressure scale height.

- a coupling between the disk temperature and its photosphere geometry (considering both the star irradiation heating (Calvet et al. 1991) and the viscous heating.

\section{Evolution and steady state}

Protoplanetary disk evolution reaches a steady state (uniform mass flux) characterized by a surface-mass density profile in $\Sigma(r) \propto r^{-1}$. Inner regions evolve much faster and outer regions keep the memory of the initial conditions much longer (Fig. 1).

\section{Results and discussion}

These results are invariant with the initial disk : the steady state does not depend on the inital disk mass or distribution for a given star. However, the evolution timescales 


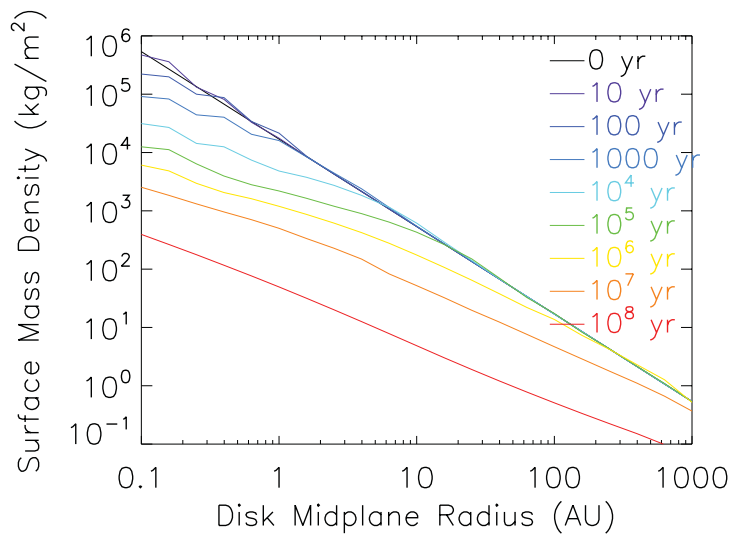

Figure 1. Evolution of the surface mass density profile.

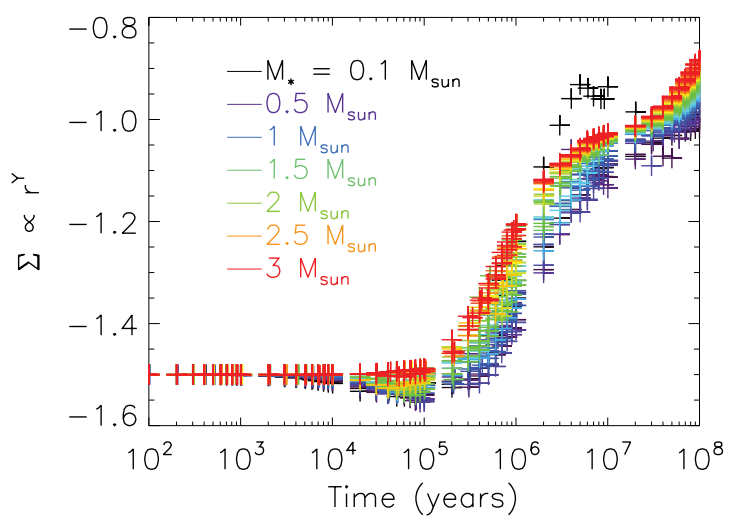

Figure 2. Temporal evolution of the surface mass density asymptotic trend for different star masses.

vary with the initial conditions : steeper or more massive initial disks will slow down the dynamical evolution and delay the reaching of the steady state while this steady state will be reached earlier for more massive stars (Fig. 2) and more turbulent disks (higher values of $\alpha_{\mathrm{visc}}$ ).

While varying independently the star mass and the disk mass, we were able to derive the following dependency for the mass accretion rate at $0.1 \mathrm{AU}$ after 1 million years: $\dot{M} \propto M_{\text {disk }} \cdot M_{*}^{0.22}$. Using observational results from Natta et al. (2006) along which $\dot{M} \propto M_{*}^{1.8}$, we predict a scaling of the disk mass along $M_{\text {disk }} \propto M_{*}^{1.6}$.

\section{References}

Andrews, S. M., Wilner, D. J., Hughes, A. M., Qi, C., \& Dullemond, C. P. 2009, ApJ, 700, 1502 Calvet, N., Patino, A., Magris, G. C., \& DAlessio, P. 1991, ApJ, 380, 617

Isella, A., Carpenter, J. M., \& Sargent, A. I. 2009, ApJ, 701, 260

Natta, A., Testi, L., \& Randich, S. 2006, A\&A, 452, 245

Shakura, N. I. \& Sunyaev, R. A. 1973, A\&A, 24,337 